\title{
Genetic Analysis of Various Agronomic Traits in Some Intra and Inter-Specific Crosses of Wheat
}

\author{
Imtiaz Ali ${ }^{1}$, Abdul Majid Khan ${ }^{1,}$ ", Maria Ayub ${ }^{1}$, Akash Zafar ${ }^{1}$, Asad-ur-Rehman ${ }^{1}$, \\ Manzoor Hussain ${ }^{1}$, Hafiz Muhammad Zia Ullah ${ }^{2}$, Muhammad Zubair ${ }^{3}$, Zeeshan Hafeez ${ }^{4}$, \\ Khalid Mahmood $^{1}$, Natasha Kanwal ${ }^{1}$, Abdul Sattar Khetran ${ }^{5}$ \\ ${ }^{1}$ Regional Agricultural Research Institute, Bahawalpur, Pakistan \\ ${ }^{2}$ Oilseeds Research Station, Khanpur, Pakistan \\ ${ }^{3}$ Agricultural Research Station, Bahawalpur, Pakistan \\ ${ }^{4}$ Oilseeds Research Station, Bahawalpur, Pakistan \\ ${ }^{5}$ Agricultural Research Institute, Quetta, Pakistan \\ Email address: \\ akhan3120@gmail.com(A. M. Khan) \\ ${ }^{*}$ Corresponding author
}

\section{To cite this article:}

Imtiaz Ali, Abdul Majid Khan, Maria Ayub, Akash Zafar, Asad-ur-Rehman, Manzoor Hussain, Hafiz Muhammad Zia Ullah, Muhammad Zubair, Zeeshan Hafeez, Khalid Mahmood, Natasha Kanwal, Abdul Sattar Khetran. Genetic Analysis of Various Agronomic Traits in Some Intra and Inter-Specific Crosses of Wheat. Plant. Vol. 6, No. 2, 2018, pp. 43-48. doi: 10.11648/j.plant.20180602.14

Received: June 12, 2018; Accepted: August 7, 2018; Published: September 6, 2018

\begin{abstract}
Six wheat genotypes (4 hexaploid and 2 tetraploid) against plant height, days to heading, days to anthesis, days to maturity, grain filling period, grains per spike and 1000 grain weight characters were studiedfor combining ability and heterosis through $6 \times 6$ half diallel fashion. This experiment was conducted at Regional Agricultural Research Institute Bahawalpur during 2016-17. Results of mean square of general combing ability (GCA) and specific combing ability (SCA) was found significant for all studied characters except forplant height and grains per spike against SCA. Ratio of GCA/SCA showed the involvement of additive gene action in the inheritance of all under studied traits. Durum-1 was found best parent among all other parents against 1000 grain weight and days to heading. Miraj-08 was good general combiner as it reflected maximum grain yield per plant. Best specific combing ability and heterosis was exhibited by cross combination Ass-11× Chakwal-50, Glaxy-13 $\times$ Chakwal-50 and Chakwal-50 $\times$ Miraj-08 against plant height, grain per spike and yield character. Results indicated that selection of best general combiner would be helpful in increasing yield through hybridization and superior hybrids may develop by the selection of superior best specific combiners.
\end{abstract}

Keywords: Triticumaestivum, General Combining Ability, Specific Combing Ability, Heterosis, Half Diallel Analysis

\section{Introduction}

Wheat is considered as important staple food and strategic crop worldwide with $27 \%$ contribution in world's cereal production. It is first ever plant grown by the human beings and due to its wider adaption and resistance to biotic and abiotic stresses it is now widely cultivated throughout the world [8].

Wheatis most important food grain crop of Pakistan. WorldwidePakistan ranked $6^{\text {th }}$ in wheat with production 25.4 million tonnes on area 9260 thousand hectares [3].
The contribution of wheat in agriculture and total GDP of Pakistan is about $9.9 \%$ and 2\% (Anonymous, 2016-17). The average wheat production has increased in Pakistan but its yield is still low when compared to many other countries. Population of Pakistan is increasing geometrically which has been a great challenge for agriculture scientists. Yield potential of recent wheat varieties is not increasing in parallel to overcome demand of population in Pakistan. To meet the challenge of increasing population there is a dire need to 
develop high yielding varieties of wheat through different breeding methods which are adaptable to different climatic condition of the country. Breeder should develop few crosses, instead of maintaining many crosses, from parents having high genetic potential. Selection of best performing parents for hybridization is based on the production of desirable segregants when a specific line combine with other lines[7]. Heterosis play important role in combing ability of parents and can be included in breeding programme [14].

Combining ability analysis is important way to select parents with desirable characters along with information regarding nature and magnitude of controlling quantitative traits [9]. Better parents are selected on the basis of general combining ability while specific combining ability is associated with the selection of superior hybrids [1]. In GCA additive genetic effect is involved while SCA is due to nonadditive genetic effect i.e dominance or epistasis. Information related to mechanism of genetic controlling different plant characters in parents may help in improving the genetic makeup of plant to maintain and improve crop production.

Diallel approach is a powerful tool for evaluating combining abilityand heterosis in additiontoanalyse the genes nature involving in the inheritance of grainyield and itscontributingtraits[11]. Parental lines can be tested in all possible combinations through diallel crosses [6]. Selection of parents with desirable characteristics showing good GCAand SCA effects for yield and its trait components are essential [4].

The objectives of present study were to estimate the combining ability of some local bread and durum wheat genotypes and to assess the heterotic potential of intra and inter-specific crosses of wheat. Another important objective was to generate diversification in wheat germplasm to be used as base line fordevelopment of highly stable wheat verieties under changing climatic conditions.

\section{Material and Methods}

Six genotypes of Triticumaestivum were crossed in half diallel fashion at Regional Agricultural Research Institute Bahawalpur during 2015-16. Spikes of female were hand emasculated for crossing and covered by butter paper bag so that contamination of foreign pollen may not occur. Pollen of selected male parent was applied on the receptive ovaries of the emasculated spikes of the female parents. Sufficient number of spikes was emasculated for each of the 6 parents and pollinated to obtain maximum seed of 15 direct crosses which were to be planted in the following year. At harvesting $\mathrm{F}_{0}$ seeds of each crosswere threshed separatelyand stored.

In the next year (2016-17) the $\mathrm{F}_{0}$ seeds of 15 crosses with six parents were sown in the field in randomized complete block design (RCBD) with three repeats. Sowing was done with manual dibbler maintainingplant to plant distance $15 \mathrm{cmand}$ row to row distance $30 \mathrm{~cm}$ respectively. In each hole two seeds were sown and later thinned to one seedling per hole after 10-12 days of germination. Standard agronomic practices were performed from sowing to maturity of crop.
At reproductive stageten guarded plants were randomly selected from each experimental unit to record data for various traits including days to heading, days to anthesis, grain filling period, days to maturity, plant height $(\mathrm{cm})$, grains per spike, 1000-grain weight (g) and grain yield per plant $(\mathrm{g})$. Combining ability analysis and heterotic effect of $\mathrm{F}_{1}$ hybrid was carried out by method given by Griffing [5].

\section{Resultsand Discussion}

\subsection{Mean Performance}

Means of parents and their cross combination were given in table 1. Broad range of expression was shown by parents for various plant characters especially for plant height ranging $88 \mathrm{~cm}$ to $102 \mathrm{~cm}$. Among six parents, minimum plant height $(88 \mathrm{~cm})$ was observed in parent Durum-2 followed by Durum-1 $(90 \mathrm{~cm})$. Genotype Ass-11 exhibited maximum plant height $102 \mathrm{~cm}$. Minimum days (94) against days to heading were recorded in Duram-2 while Chakwal-50 showed maximum days in heading i.e 102. Genotype Duram-2 took 100 days to anthesis while maximum days were recorded in glaxy-13 (109). Early maturity (139 days) was observed in Miraj-08 followed by Duram-1 in which 142 days were recorded for maturity. Glaxy-13 showed maximum days i.e 148 to mature. Genotype Miraj-08 exhibited minimum days (36) against grain filling character and maximum days (43) for grain filling was recorded in Duram-2. Maximum no of grain per spike shown by Ass-11 which was 54 followed by Chakwal-50 in which 50 grain/spike was recorded. Ass-11 and Duram-1 showed maximum weight (42g) against 1000 grain weight and minimum weight was observed in Chakwal50 which was $39 \mathrm{~g}$. Highest grain yield/plant $(35 \mathrm{~g})$ was observed in variety Glaxy-13 followed by Miraj- 08 which exhibited $34 \mathrm{~g}$ yield/plant while Duram-1 and Duram-2 showed minimum yield/ plant $31 \mathrm{~g}$ among all parents.

Mean values of 15 crosses were presented in table 1 . Cross combination Durum-1 x Durum-2 performed better against plant height trait with minimum value $90 \mathrm{~cm}$ followed by cross Miraj-08 x Durum-1 having plant height $92 \mathrm{~cm}$. Better performance with minimumvalue (94) for days to heading was performed by cross Ass-11 x Miraj-08 and maximum value was recorded for Ass-11 x Chakwal-50. Glaxy-13 x Durum-2 took 99 days for anthesis while maximum days i.e 109 was recorded for Glaxy-13 x Ass-1. Early maturity (140 days) was recorded in cross Miraj-08 x Durum-1and cross combination Miraj-08 x Durum-2 took minimum days (32) for grain filling while maximum days was exhibited by Glaxy-13 x Durum-2 which was 45 days. Highest grains (50) in a spike was found in cross Ass-11 x Durum-2 followed by Chakwal-50 x Durum-2 (49). Weight of 1000-grain is a vital yield component and is more or less stable character of wheat. Cross combination Durum-1 x Durum-2 and Chakwal-50 x Miraj-09 exhibited maximum 1000 grain weight $44 \mathrm{~g}$ and yield per plant $37 \mathrm{~g}$. 
Table 1. Mean values of parents and crosses for yield related traits in wheat.

\begin{tabular}{lllllllll}
\hline & $\begin{array}{l}\text { Plant } \\
\text { height }\end{array}$ & $\begin{array}{l}\text { Days to } \\
\text { heading }\end{array}$ & $\begin{array}{l}\text { Days to } \\
\text { anthesis }\end{array}$ & $\begin{array}{l}\text { Days to } \\
\text { maturity }\end{array}$ & $\begin{array}{l}\text { Grain filling } \\
\text { period }\end{array}$ & $\begin{array}{l}\text { Grains per } \\
\text { spike }\end{array}$ & $\begin{array}{l}\text { 1000-grain } \\
\text { weight }\end{array}$ & $\begin{array}{l}\text { Grain yield } \\
\text { per plant }\end{array}$ \\
\hline Parents & & & & & & & & \\
Galaxy-13 & 101 & 101 & 109 & 148 & 39 & 46 & 39 & 35 \\
Aas-11 & 102 & 101 & 108 & 144 & 37 & 54 & 42 & 33 \\
Chakwal-50 & 98 & 102 & 107 & 146 & 39 & 50 & 39 & 33 \\
Mairaj-08 & 93 & 95 & 103 & 139 & 36 & 46 & 40 & 34 \\
Durum-1 & 90 & 96 & 102 & 142 & 39 & 44 & 42 & 31 \\
Durum-2 & 88 & 94 & 100 & 143 & 43 & 49 & 38 & 31 \\
Crosses & & & & & & & \\
Galaxy-13 $\times$ Aas-11 & 102 & 100 & 109 & 146 & 37 & 49 & 39 & 36 \\
Galaxy-13 $\times$ Chakwal-50 & 100 & 99 & 106 & 145 & 38 & 48 & 38 & 36 \\
Galaxy-13 $\times$ Mairaj-08 & 97 & 96 & 102 & 141 & 39 & 46 & 40 & 34 \\
Galaxy-13 $\times$ Durum-1 & 96 & 100 & 105 & 144 & 39 & 45 & 39 & 33 \\
Galaxy-13 $\times$ Durum-2 & 96 & 95 & 99 & 145 & 45 & 47 & 37 & 34 \\
Aas-11 $\times$ Chakwal-50 & 99 & 102 & 109 & 144 & 35 & 50 & 40 & 35 \\
Aas-11 $\times$ Mairaj-08 & 100 & 94 & 101 & 142 & 41 & 49 & 41 & 37 \\
Aas-11 $\times$ Durum-1 & 101 & 100 & 107 & 142 & 36 & 48 & 40 & 33 \\
Aas-11 $\times$ Durum-2 & 101 & 101 & 108 & 144 & 36 & 50 & 38 & 32 \\
Chakwal-50 $\times$ Mairaj-08 & 97 & 96 & 101 & 142 & 41 & 46 & 43 & 37 \\
Chakwal-50 $\times$ Durum-1 & 94 & 102 & 108 & 144 & 36 & 45 & 41 \\
Chakwal-50 $\times$ Durum-2 & 94 & 98 & 105 & 145 & 40 & 49 & 37 \\
Mairaj-08 $\times$ Durum-1 & 92 & 99 & 106 & 140 & 34 & 44 & 41 & 33 \\
Mairaj-08 $\times$ Durum-2 & 93 & 102 & 108 & 140 & 32 & 47 & 38 \\
Durum-1 $\times$ Durum-2 & 90 & 95 & 101 & 141 & 40 & 46 & 44 \\
\hline
\end{tabular}

\subsection{Combining Ability}

\subsubsection{Analysis of Variance (ANOVA)}

Plant breeders rely on the availability of genetic diversityfor the improvement of any plant trait. Significant differences (Table 2) of wheat varieties and their hybrids indicated the presence of genetic variation between genotypes and crosses. Highly significant or significant mean squares due to male, female and their interaction showed the involvement ofboth additive and non-additive i.e dominance and epistasis gene action in the expression of studied triats. [2] also found significant differences among wheat varieties against days to heading, weight of 1000 grain and grain yield per plant. General combing ability (GCA) of parents was observed higher than specific combing ability (SCA) of crosses for all traits like plant height, days to heading, days to anthesis, days to maturity, grain filling period, grains per spike, 1000 grain weight and grain yield per plant. High GCA reflected the prominent role of additive type of gene action in the inheritance of studied traits although significant mean squares for specific combing ability of crosses werealso observed against characters except plant height and grains per spike. Similar findings have also been observed in wheat by [12].

Table 2. Mean square values corresponding to diallel analysis for various characters in bread wheat.

\begin{tabular}{|c|c|c|c|c|c|c|c|c|c|}
\hline SOV & d.f. & Plant height & $\begin{array}{l}\text { Days to } \\
\text { heading }\end{array}$ & $\begin{array}{l}\text { Days to } \\
\text { anthesis }\end{array}$ & $\begin{array}{l}\text { Days to } \\
\text { maturity }\end{array}$ & $\begin{array}{l}\text { Grain filling } \\
\text { period }\end{array}$ & $\begin{array}{l}\text { Grains per } \\
\text { spike }\end{array}$ & $\begin{array}{l}\text { 1000-grain } \\
\text { weight }\end{array}$ & $\begin{array}{l}\text { Grain yield } \\
\text { per plant }\end{array}$ \\
\hline Replication & 2 & $3.1027^{\mathrm{NS}}$ & $5.9141^{\mathrm{NS}}$ & $15.0714^{\mathrm{NS}}$ & $10.3542 * *$ & $0.4449^{\mathrm{NS}}$ & $27.4464^{* *}$ & $1.1594^{\mathrm{NS}}$ & $1.0173^{\mathrm{NS}}$ \\
\hline Genotype & 20 & $6.4949 * *$ & $25.1385 * *$ & $34.0833 * *$ & $15.7354 * *$ & $29.530 * *$ & $17.7208 * *$ & $10.5207 * *$ & $11.0493 * *$ \\
\hline GCA & 5 & $61.20 * *$ & $11.53^{* *}$ & $15.33^{* *}$ & $18.75^{* *}$ & $10.15^{*}$ & $22.03 * *$ & $6.75 * *$ & $8.29 * *$ \\
\hline SCA & 15 & 2.99 & $1.97 * *$ & $10.03 * *$ & $0.74 *$ & $9.73 * *$ & 0.53 & $2.42 *$ & $2.14^{*}$ \\
\hline Error & 40 & 2.16 & 7.32 & 3.02 & 0.34 & 3.00 & 1.63 & 1.18 & 1.01 \\
\hline
\end{tabular}

\subsubsection{General Combing Ability}

The general combing ability gives information about better performing parents based on their hybrid performance to exploit heterosis breeding. Among parents, genotype Durum2 was best general combiner against plant height and days to anthesis with minimum negative value 2.93 and 1.74 . Durum-1 exhibited maximum 1000 grain weight (1.14). Minimum days to heading (1.38), days to maturity (2.38) and maximum grain yield per plant (1.06) was shown by Miraj08. Ass-11 showedbest performance against grain filling period character with minimum negative value 1.22 and exhibited maximum no of grains per spike 2.61. Parents with maximum GCA effects were the best general combiners as valuable genes were present in them for the improvement of traits and such parents may be preferred for selection and hybrid development [10].

\subsubsection{Specific Combing Ability}

Among fifteen hybrids, best specific combing ability against plant height was exhibited by cross combination Ass$11 \mathrm{x}$ Chakwal-50 with minimum negative value $1.91 \mathrm{~cm}$. 
Minimum days to heading (4.14) and days to anthesis (4.68) was recorded in Ass-11 x Miraj-08. The hybrid Glaxy-13 x Chakwal-50 was find to be best specific combiner against days to maturity and grains per spike with minimum negative value 1.61 and maximum positive value 0.58 . Minimum days in grain filling period was recorded in cross Miraj-08 $\mathrm{x}$ Durum-2 as it showed minimum negative SCA value 7.10. High yield per plant (2.26g) was observed in cross combination Chakwal-50 x Miraj-08 (Table 3). Selection of such crosses with high specific combing abilitiy would be fruitful in the development of superior hybrid through heterosis breeding. It is not a rule that hybrid should be produced by parents with best general combiners sometimes hybrid may be developed by the combination of either parent showing poor GCA (Patel et al., 1997)e.g Ass-11 x Chakwal50 for plant height, Ass-11 x Miraj-08 for days to anthesis, Glaxy-13 x Chakwal-50 for days to maturity and Durum-1 x Durum-2 for 1000 grain weight respectively in this study.

Table 3. Estimates of GCA effects of parents and SCA effects of crosses for yield related characters of wheat.

\begin{tabular}{|c|c|c|c|c|c|c|c|c|}
\hline & $\begin{array}{l}\text { Plant } \\
\text { height }\end{array}$ & $\begin{array}{l}\text { Days to } \\
\text { heading }\end{array}$ & $\begin{array}{l}\text { Days to } \\
\text { anthesis }\end{array}$ & $\begin{array}{l}\text { Days to } \\
\text { maturity }\end{array}$ & $\begin{array}{l}\text { Grain filling } \\
\text { period }\end{array}$ & $\begin{array}{l}\text { Grains per } \\
\text { spike }\end{array}$ & $\begin{array}{l}\text { 1000-grain } \\
\text { weight }\end{array}$ & $\begin{array}{l}\text { Grain yield } \\
\text { per plant }\end{array}$ \\
\hline \multicolumn{9}{|l|}{ GCA for parents } \\
\hline Galaxy-13 & $2.36 * *$ & 0.17 & 0.81 & $1.87 * *$ & 1.07 & -0.68 & $-0.90 *$ & $0.85 *$ \\
\hline Aas-11 & $3.82 * *$ & $1.08 *$ & $1.72 * *$ & $0.50 *$ & $-1.22 *$ & $2.61 * *$ & 0.47 & 0.35 \\
\hline Chakwal-50 & 0.69 & $1.58 * *$ & 0.97 & $1.17 * *$ & 0.19 & 0.61 & -0.07 & 0.22 \\
\hline Mairaj-08 & $-1.18 *$ & $-1.38 * *$ & $-1.36 *$ & $-2.38 * *$ & -1.01 & $-1.06 *$ & 0.60 & $1.06 * *$ \\
\hline Durum-1 & $-2.76 * *$ & -0.21 & -0.40 & $-0.96 * *$ & -0.56 & $-2.14 * *$ & $1.14 * *$ & $-0.99 * *$ \\
\hline \multicolumn{9}{|l|}{ SCA for crosses } \\
\hline Galaxy-13 × Aas-11 & -0.91 & $-0.01 \mathrm{~ns}$ & $1.82 *$ & 0.39 & -1.43 & -0.42 & -0.08 & $1.35 * *$ \\
\hline Galaxy-13 $\times$ Chakwal-50 & 0.21 & $-1.51 *$ & -0.43 & $-1.61 * *$ & -1.18 & 0.58 & -0.54 & $1.14 *$ \\
\hline Galaxy-13 × Mairaj-08 & -0.58 & $-1.22 *$ & $-2.10 * *$ & $-1.40 * *$ & 0.69 & -0.09 & 0.80 & $-1.70 * *$ \\
\hline Galaxy-13 × Durum-1 & 0.34 & $1.28 *$ & -0.05 & 0.18 & 0.23 & 0.33 & $-1.41 * *$ & $-0.99 *$ \\
\hline Galaxy-13 × Durum-2 & 0.51 & $-2.35 * *$ & $-4.72 * *$ & -0.24 & $4.48 * *$ & -0.13 & -0.70 & $1.18 * *$ \\
\hline Aas-11 × Chakwal-50 & $-1.91 * *$ & $1.24 *$ & 1.32 & $-0.57 *$ & $-1.89 *$ & -0.71 & 0.09 & 0.64 \\
\hline Aas-11 × Durum-2 & $3.38 * *$ & $2.40 * *$ & $3.36 * *$ & $0.80 * *$ & $-2.56 * *$ & -0.76 & $-1.41 * *$ & $-0.99 *$ \\
\hline Chakwal-50 $\times$ Mairaj-08 & $1.42 *$ & $-2.30 * *$ & $-3.26 * *$ & 0.30 & $3.57 * *$ & -1.05 & $2.63 * *$ & $2.26 * *$ \\
\hline Chakwal-50 × Durum-1 & 0.01 & $1.86 * *$ & $2.45 * *$ & 0.22 & $-2.23 * *$ & $-1.30 *$ & 0.09 & -0.36 \\
\hline Chakwal-50 × Durum-2 & -0.16 & -0.76 & 0.45 & $0.80 * *$ & 0.36 & -0.09 & $-1.54 * *$ & -0.53 \\
\hline Mairaj-08 × Durum-1 & -0.79 & $2.49 * *$ & $2.78 * *$ & 0.43 & $-2.35 * *$ & -0.30 & -0.58 & $1.80 * *$ \\
\hline Mairaj-08 × Durum-2 & 1.05 & $6.53 * *$ & $6.45 * *$ & $-0.65 *$ & $-7.10 * *$ & 0.24 & -0.87 & 0.30 \\
\hline Durum-1 × Durum-2 & -0.37 & $-1.97 * *$ & $-1.85 *$ & $-1.07 * *$ & 0.77 & 0.33 & $3.92 * *$ & 0.35 \\
\hline
\end{tabular}

\subsubsection{Heterosis}

Means of eight characters of fifteen $F_{1}$ hybrids were compared with values of mid parent (relative heterosis), better parent (heterobeltosis) and over standard parent (table 4).

Short stature and early maturing plants of wheat are required to improve plant height and yield improving characters, hence negative heterosis is favourable for plant height, days to heading, days to anthesis, days to maturity and grain filling period. Heterosis for plant height was nonsignificant in all crosses. Maximum heterosis over Mid parent was exhibited by cross combinations Ass-11 $\times$ Chakwal-50 (-0.835cm), Ass-11 × Miraj-08 (-3.918), Glaxy$13 \times$ Durum-2 (-4.944), Glaxy-13 × Chakwal-50 (-1.699), Miraj-08 × Durum-2 (-20.50g), Durum-1 × Durum-2 (10.084g), and Chakwal-50 $\times$ Miraj-08 (12.562) against plant height, days to heading, days to anthesis, days to maturity, grain filling period, 1000 grain weight and yield/plant.

Highly significant negative heterobeltosis was found in Glaxy-13 × Durum-2 (-9.146)against days to heading and plant height $(-4.934 \mathrm{~cm})$ while Chakwal-50 $\times$ Miraj-08 showed positive heterotic effect i.e $7.500 \mathrm{~g}$ and $10.891 \mathrm{~g}$ for thousand grain weight and yield/plant relative to better parent heterosis. Minimum day to heading (-6.623) and days to maturity (-4.719) was exhibited by Ass-11 $\times$ Miraj-08 and Glaxy-13 $\times$ Miraj-08. Minimum heterobeltosis for grain filling period was shown by hybrid Miraj-08 $\times$ Durum-2 (26.923).

Significant positive results 8.695 and $6.666 \mathrm{~g}$ relative to standard heterosis were recorded for cross combination Ass$11 \times$ Chakwal-50 and Chakwal-50 $\times$ Miraj-08 for grain/spike and yield/plant. Durum-1 $\times$ Durum-2 reflected short stature ($10.855 \mathrm{~cm})$ and Ass-11 $\times$ Miraj-08 required minimum days to heading (-6.623). Early maturing characters like days to anthesis(-9.146) was observed by hybrid Glaxy-13 $\times$ durum2 while minimum days for grain filling $(-18.803)$ period and days to maturity (-5.617) were shown by Miraj-08 $\times$ Durum2 . These hybrids contained valuable genes from both parents and thus grain yield and yield contributing traits can be increased by exploring hybrids which exhibited maximum heterosis and heterobeltosis under studied traits [13]. 
Table 4. Heterotic effects of F1 hybrids over mid parents (MPH) and better parents (BPH) and standard heterosis (STH).

\begin{tabular}{|c|c|c|c|c|c|c|c|c|c|}
\hline Crosses & & $\begin{array}{l}\text { Plant } \\
\text { height }\end{array}$ & $\begin{array}{l}\text { Days to } \\
\text { heading }\end{array}$ & $\begin{array}{l}\text { Days to } \\
\text { anthesis }\end{array}$ & $\begin{array}{l}\text { Days to } \\
\text { maturity }\end{array}$ & $\begin{array}{l}\text { Grain } \\
\text { filling } \\
\text { period }\end{array}$ & $\begin{array}{l}\text { Grains } \\
\text { per spike }\end{array}$ & $\begin{array}{l}\text { 1000- } \\
\text { grain } \\
\text { weight }\end{array}$ & $\begin{array}{l}\text { Grain } \\
\text { yield per } \\
\text { plant }\end{array}$ \\
\hline \multirow{3}{*}{ Galaxy-13 × Aas-11 } & $\mathrm{MPH}$ & 0.164 & -0.993 & 0.923 & -0.114 & -3.083 & -2.000 & -2.880 & $6.8627 *$ \\
\hline & $\mathrm{BPH}$ & 0.000 & -0.993 & 0.000 & $-1.573 *$ & -5.982 & $-9.259 *$ & -6.349 & 3.810 \\
\hline & STH & 0.329 & -0.993 & 0.000 & $-1.573 *$ & -5.982 & 6.521 & 0.855 & 3.810 \\
\hline \multirow{3}{*}{ Glaxy-13 × Chakwal-50 } & $\mathrm{MPH}$ & 0.000 & -2.791 & -1.543 & $-1.699 * *$ & -2.127 & 0.000 & -2.127 & $6.4039 *$ \\
\hline & $\mathrm{BPH}$ & -1.645 & -3.583 & -2.743 & $-2.472 * *$ & -2.542 & -4.000 & -2.542 & 2.857 \\
\hline & STH & -1.645 & -1.987 & -2.743 & $-2.472 * *$ & -1.709 & 4.347 & -1.709 & 2.857 \\
\hline \multirow{3}{*}{ Galaxy-13 × Mairaj-08 } & $\mathrm{MPH}$ & -0.172 & -1.874 & $-3.459 *$ & $-1.624^{* *}$ & 3.539 & 0.000 & 2.109 & -0.971 \\
\hline & $\mathrm{BPH}$ & -4.276 & -4.636 & $-6.402 *$ & $-4.719 * *$ & 0.000 & 0.000 & 0.833 & -2.857 \\
\hline & STH & -4.276 & -4.636 & $-6.402 *$ & $-4.719 * *$ & 0.000 & 0.000 & 3.418 & -2.857 \\
\hline \multirow{3}{*}{ Galaxy-13 × Durum-1 } & $\mathrm{MPH}$ & 0.873 & 1.356 & -0.472 & -0.460 & -0.425 & 0.000 & -4.132 & -1.507 \\
\hline & $\mathrm{BPH}$ & -4.934 & -0.993 & -3.658 & $-2.696 * *$ & -0.847 & -2.173 & -7.200 & -6.667 \\
\hline & STH & -4.934 & -0.993 & -3.658 & $-2.696 * *$ & 0.000 & -2.173 & -0.854 & -6.667 \\
\hline \multirow{3}{*}{ Galaxy-13 × Durum-2 } & MPH & 1.582 & -2.397 & $-4.944 *$ & -0.686 & $10.121^{*}$ & -1.052 & -3.478 & 4.569 \\
\hline & $\mathrm{BPH}$ & -4.934 & -5.629 & $-9.146 * *$ & $-2.471 * *$ & 4.615 & -4.081 & -5.128 & -1.905 \\
\hline & STH & -4.934 & -5.629 & $-9.146 * *$ & $-2.471 * *$ & $16.239 *$ & 2.173 & -5.128 & -1.905 \\
\hline \multirow{3}{*}{ Aas-11 $\times$ Chakwal-50 } & MPH & -0.835 & 0.821 & 1.869 & -0.459 & -7.017 & -3.846 & -0.819 & $6.5989 *$ \\
\hline & $\mathrm{BPH}$ & -2.623 & 0.000 & 1.552 & -1.141 & -10.169 & $-7.407 *$ & -3.968 & 6.061 \\
\hline & STH & -2.303 & 1.656 & -0.304 & $-2.696 * *$ & -9.401 & $8.695 *$ & 3.418 & 0.000 \\
\hline \multirow{3}{*}{ Aas-11 $\times$ Mairaj-08 } & $\mathrm{MPH}$ & 2.740 & -3.918 & $-4.126^{*}$ & 0.117 & $12.328 *$ & -2.000 & 0.000 & $11 * *$ \\
\hline & $\mathrm{BPH}$ & -1.639 & -6.623 & $-6.211 *$ & $-1.620 *$ & 11.818 & $-9.259 *$ & -2.380 & $9.9009^{*}$ \\
\hline & STH & -1.316 & -6.623 & $-7.926 * *$ & $-4.494 * *$ & 5.128 & 6.521 & 5.128 & 5.714 \\
\hline \multirow{3}{*}{ Aas-11 × Durum-1 } & MPH & 5.226 & 1.356 & 1.748 & -0.350 & -6.140 & -2.040 & -3.585 & 3.627 \\
\hline & BPH & -0.984 & -0.993 & -0.621 & -1.157 & -9.322 & $-11.11 * *$ & -3.968 & 1.010 \\
\hline & STH & -0.658 & -0.993 & -2.439 & $-4.044 * *$ & -8.547 & 4.347 & 3.418 & -4.762 \\
\hline \multirow{3}{*}{ Aas-11 × Durum-2 } & MPH & 5.965 & 3.425 & $4.669 *$ & 0.580 & $-10 *$ & -2.912 & -5.439 & -0.523 \\
\hline & $\mathrm{BPH}$ & -0.984 & 0.000 & 0.931 & 0.231 & $-16.92 * *$ & $-7.407 *$ & $-10.31 *$ & -4.040 \\
\hline & STH & -0.658 & 0.000 & -0.914 & $-2.696 * *$ & -7.692 & $8.695 *$ & -3.418 & $-9.523 *$ \\
\hline \multirow{3}{*}{ Chakwal-50 $\times$ Mairaj-08 } & $\mathrm{MPH}$ & 1.920 & -2.365 & -3.184 & -0.116 & 8.370 & -4.166 & $8.403 * *$ & $12.562 * *$ \\
\hline & $\mathrm{BPH}$ & -0.680 & -5.863 & $-5.00 *$ & $-2.511 * *$ & 4.237 & $-8^{*}$ & 7.500 & $10.891 *$ \\
\hline & STH & -3.947 & -4.305 & $-7.317 * *$ & $-4.044 * *$ & 5.128 & 0.000 & $10.256^{*}$ & 6.666 \\
\hline \multirow{3}{*}{ Chakwal-50 × Durum-1 } & $\mathrm{MPH}$ & 0.533 & 2.521 & 3.349 & -0.115 & $-9.322 *$ & -4.255 & 1.235 & 2.083 \\
\hline & $\mathrm{BPH}$ & -3.742 & -0.651 & 1.250 & $-1.598^{*}$ & -9.322 & $-10^{*}$ & -1.600 & 0.000 \\
\hline & STH & -6.908 & 0.993 & -1.219 & $-3.146^{* *}$ & -8.547 & -2.173 & 5.128 & -6.666 \\
\hline \multirow{3}{*}{ Chakwal-50 × Durum-2 } & $\mathrm{MPH}$ & 0.894 & -0.170 & 1.453 & 0.346 & -2.419 & -1.010 & -3.896 & 1.052 \\
\hline & $\mathrm{BPH}$ & -4.082 & -4.235 & -1.875 & -0.684 & -6.923 & -2.000 & -1.769 & -2.040 \\
\hline & STH & -7.237 & -2.649 & -4.268 & $-2.247 * *$ & 3.418 & 6.521 & -5.128 & $-8.571 *$ \\
\hline \multirow{3}{*}{ Mairaj-08 × Durum-1 } & MPH & 0.365 & 4.014 & 3.414 & 0.000 & -9.251 & -2.222 & 0.408 & $9.743 * *$ \\
\hline & BPH & -1.434 & 3.472 & 3.246 & -0.941 & -12.711 & -4.347 & -1.600 & 5.940 \\
\hline & STH & -9.539 & -1.325 & -3.048 & $-5.393 * *$ & -11.965 & -4.347 & 5.128 & 1.904 \\
\hline \multirow{3}{*}{ Mairaj-08 $\times$ Durum-2 } & $\mathrm{MPH}$ & 2.941 & $8.289242 *$ & $7.084^{* *}$ & -0.709 & $-20.50 * *$ & -1.052 & -1.287 & 4.663 \\
\hline & $\mathrm{BPH}$ & 0.358 & $7.719298 *$ & $5.519 *$ & $-2.097 * *$ & $-26.923 * *$ & -4.081 & -4.166 & 0.000 \\
\hline & STH & -7.895 & 1.656 & -0.914 & $-5.617 * *$ & $-18.803 * *$ & 2.173 & -1.709 & -3.809 \\
\hline \multirow{3}{*}{ Durum-1 $\times$ Durum-2 } & MPH & 1.498 & 0.000 & 0.000 & $-0.936^{*}$ & -3.226 & -1.075 & $10.084 * *$ & 2.150 \\
\hline & BPH & 0.743 & -1.042 & -1.302 & $-1.398 *$ & -7.692 & -6.122 & 4.800 & 1.063 \\
\hline & STH & -10.855 & -5.629 & $-7.621 *$ & $-4.943 * *$ & 2.564 & 0.000 & $11.965 * *$ & $-9.523 *$ \\
\hline
\end{tabular}

\section{Conclusion}

Our results indicated the presence of both additive and non additive (dominant or epistasis) gene action in the inheritance of studied traits. General combing ability (GCA) of parents was observed higher than specific combing ability (SCA) of crosses for all traits like plant height, days to heading, days to anthesis, days to maturity, grain filling period, grains per spike, 1000 grain weight and grain yield per plant. Durum-1 was found best parent among all other parents against 1000 grain weight and days to heading. Miraj-08 was good general combiner as it reflected maximum grain yield per plant. These parents contain valuable genes for improvement of characters and may be preffered for selection and hybridization program. Best specific combing ability and heterosis was exhibited by cross combination Ass-11× Chakwal-50, Glaxy-13 $\times$ Chakwal-50 and Chakwal-50 $\times$ Miraj-08 against plant height, grain per spike and yield character. Selection of such crosses would be beneficial for the development of superior hybrids through heterosis breeding. 


\section{References}

[1] Ahuja SL \&Dhayal LS, 2007. Combining ability estimates for yield and fibre quality traits in $4 \times 13$ line $\mathrm{x}$ tester crosses of Gossypiumhirsutum. Euphytica. 153:87-98.

[2] Akinci C, 2009. Heterosis and combining ability estimates in $6 \times 6$ halfdiallel crosses of durumwheat (Triticum durum desf.). Bulgarian. J. Agric. Sci. 15 (3): 214-221.

[3] Anonymous. 2016-17. Pakistan Economic Survey. Ministry of Finance, Economic Advisor's Wing, Islamabad.

[4] DesaleCS, Mehta DR\&Singh AP, 2014. Combining ability analysis in bread wheat. J. Wheat Res. 6: 25-28.

[5] Griffing B, 1956. Concept of general and specific combining ability in relation to diallelcrossing system. Aust. J. Biol. Sci. 9: 463-493.

[6] HassanG, Mohammad F, Afridi SS \&Khalil I, 2007. Combining ability in the F1 generationsof diallel crosses for yield and yield components in wheat. Sarhad. J. Agric. 23: 937-942.

[7] Jaiswal, KK, Marker S \& Kumar B, 2013. Combining ability analysis in diallel crosses ofwheat (Triticumaestivum L.). The Bioscan. 8: 1557-1560.

[8] Khayatnejad M, Zaefizadeh M \&Gholamin R, 2010. Study of genetic diversity and pathanalysis for yield of durum wheat under drought stress condition. Plant Ecophysiol. 2: 133-136.

[9] MasoodSA, Ahmad S, Kashif M and Ali Q, 2014. Role of combining ability to develop higheryielding wheat (Triticumaestivum L.) genotypes: An overview. Natural Sciences. 12: 155-161.

[10] Peng Z, Ru PH, Fu NZ, Hu LL, Jun W \&Xin SQ, 2009. Heterosis and combining ability ofmajor agronomic traits in crosses between earbranched wheat and common wheat. J. TriticeaeCrops. 29: 212216.

[11] Rashid M, Cheema AA \& Ashraf M, 2007. Line $\times$ tester analysis in basmati rice. Pakistan J. Bot. 39(6): 2035-2042.

[12] Sharma, P. K \& Pawar IS, 2000. Genetic architecture of some wheat crosses through triple testcross method. Ind. J. Genet Plant Breed. 48:45-48.

[13] SharmaSK, Singh KP \&Singh I, 1991. Selection responses for grain weight in someintermated population of wheat (Triticumaestivum L.). Proc. Golden Jubilee Symp. IndianSoc. Genet. Plant Breed. New Delhi. Abstr. II: 357.

[14] Singh M, Devi EL, Glawe S, Kousar N \&Behera C, 2013. Estimation of heterosis in different crosses of bread at (Triticumaestivum L.). The Bioscan. 8: 1393-1401. 